\title{
Correlation between Primary Myelofibrosis and the Association of Portal Thrombosis with Portal-Biliary Cavernoma: US, MDCT, and MRI Features
}

\author{
Marco Di Girolamo ${ }^{1}$ Stefania Galassi ${ }^{1} \quad$ Salvatore Merola ${ }^{1}$ \\ ${ }^{1}$ Department of Radiology, Faculty of Medicine and Psychology, \\ Sapienza University of Rome, Sant'Andrea Hospital, Rome, Italy \\ 2Department of Hemathology, Faculty of Medicine and Psychology, \\ Sapienza University of Rome, Sant’Andrea Hospital, Rome, Italy
}

Paolo Bonome ${ }^{1}$

Esmeralda Conte ${ }^{2}$
Elsa lannicelli ${ }^{1}$
Address for correspondence Marco Di Girolamo, MD, Department of Radiology, Faculty of Medicine and Psychology, Sapienza University of Rome, Sant'Andrea Hospital, Via di Grottarossa 1035, 00189 Roma, Italy (e-mail: marcodigiro@hotmail.com).

J Gastrointestinal Abdominal Radiol ISGAR 2021;4:8-13.

\begin{abstract}
Keywords

- primary myelofibrosis

- portal system

- thrombosis

- portal-biliary cavernoma

- diagnostic imaging

- ultrasonography

Objective Myelofibrosis is a rare chronic myelolymphoproliferative disease and is associated with increased risk of venous thromboembolism. The objective of this study is to retrospectively evaluate patients with primary myelofibrosis who underwent abdominal US, MDCT and MRI, in order to identify the development of portal thrombosis and its correlation with portal-biliary cavernoma.

Methods We evaluated 125 patients with initial diagnosis of primary myelofibrosis and nonspecific abdominal pain who had undergone US with color Doppler. In 13 patients ( 8 men, 5 females; age: $45-85$ ), US detected portal thrombosis with associated portal-biliary cavernoma. All patients subsequently underwent contrast-enhanced MDCT and MRI and 4 patients MR-cholangiography. The correlation between primary myelofibrosis and portal thrombosis and cavernoma respectively was calculated using $\chi^{2}$ test.

Results About $10 \%$ of patients with primary myelofibrosis preliminary evaluated with US had partial ( 8 pts) or complete ( 5 pts) portal thrombosis associated with portal-biliary cavernoma with a $\chi^{2}=0$. In all patients, US detected a concentric thickening of main bile duct (MBD) wall (mean value: $7 \mathrm{~mm}$ ); color Doppler always showed dilated venous vessels within the thickened wall of the biliary tract. Contrast-enhanced CT and MRI confirmed thickening of MBD walls with their progressive enhancement and allowed better assessment of the extent of the portal system thrombosis. MR-cholangiography showed a thin appearance of the MBD lumen with evidence of ab extrinsic compression. Conclusions The evidence of portal thrombosis and portal-biliary cavernoma in $10 \%$ of the patients with primary myelofibrosis indicates a close correlation between the two diseases. In the detection of portal thrombosis and portal-biliary cavernoma, US with color Doppler is the most reliable and economical diagnostic technique while contrast-enhanced MDCT and MRI allow better assessment of the extent of the portal vein thrombosis and of the complications of myelofibrosis.
\end{abstract}

\section{Introduction}

The portal cavernoma and more uncommon biliary cavernomatosis are pathologies characterized by formation of hepatopetal collateral venous circles in the context of the wall of these two

published online October 12, 2020
DOI https://doi.org/ 10.1055/s-0040-1716606 ISSN 2581-9933. anatomical structures, and in most cases, they are due to a progressive obstruction of an extensive portion of the portal vein. ${ }^{1-3}$

Biliary involvement in cavernomatous transformation, also called biliary cavernomatosis or portal biliopathy, is a pathological condition which has witnessed a growing 
interest. The first cases were reported in 1961 by Saint ${ }^{4}$ and in 1965 by Hunt. ${ }^{5}$ Saint first described the surgical and histopathological aspect of a venous plexus in close contact with the wall of the main bile duct (MBD) and suggested the possibility that this anomaly could be related to the presence of some specific liver pathologies.

For a long time, the cavernous transformation of the biliary tract has been considered a very rare and unusual condition ${ }^{3,6,7}$; Novellas et $\mathrm{al}^{6}$ accidently found this pathological entity with relative greater frequency during the execution of some diagnostic imaging techniques, more frequently in the course of endoscopic retrograde cholangiopancreatography (ERCP) and MR-cholangiography.

This condition is still poorly known and easily confused with other causes of parietal thickening of the MBD. Thus, this diagnosis is fundamental for an appropriate therapeutic treatment, which is reserved exclusively for symptomatic cases ${ }^{8,9}$ and is important to avoid the onset of potentially life-threatening complications, such as massive bleeding, during the execution of some procedures such as ECRP and choledocholithotomy. ${ }^{6,10}$

Previous studies have shown the wall of the biliary tract affected by cavernomatosis have a thickness ranging from 5 to $6 \mathrm{~mm}^{7}$

Portal and biliary cavernomatosis is caused by portal hypertension, seen in $40 \%$ of cases with extrahepatic obstruction of the portal vein (EHOPV). ${ }^{11}$ The most common causes of obstruction of the portal vein in adults include thrombosis, malignant tumors, trauma, and inflammatory abdominal diseases. ${ }^{12}$

In 40 to $70 \%$ of patients without liver cirrhosis or hepatocellular carcinoma, portal thrombosis is related to the presence of hereditary or acquired thrombophilic factors and blood hypercoagulability that increases probability of thrombi formation in splanchnic veins ${ }^{13-15}$ (-Table 1). Acquired states of hypercoagulability include oral contraceptives, pregnancy, inflammatory states and myelolymphoproliferative diseases. ${ }^{16}$

Myelofibrosis is a rare chronic myelolymphoproliferative disease characterized by megakaryocyte and myeloid proliferation, reactive medullary fibrosis, ineffective erythropoiesis and extramedullary hematopoiesis. ${ }^{17,18}$

The aim of this work was to retrospectively evaluate patients with initial diagnosis of primary myelofibrosis who had undergone abdominal ultrasound with color Doppler and subsequent multidetector CT, abdominal MRI with the administration of contrast media (MDC) and MR-cholangiography to identify portal thrombosis and the presence of portobiliary cavernomatosis.

\section{Materials and Methods}

The proposed work is a retrospective study. The completed study was performed in accordance with the ethical standards expressed by the 1964 Helsinki Declaration. Patients admitted in our institution signed a written informed consent, which allows us to use patients' data for educational and research purposes. Furthermore, the criteria of anonymity and confidentiality of the sensitive data of the enrolled patients was followed in the retrospective evaluation. No
Table 1 Acquired or hereditary conditions that determine hypercoagulable conditions

\begin{tabular}{|l|l|}
\hline Acquired conditions & $\begin{array}{l}\text { Hereditary or congenital } \\
\text { conditions }\end{array}$ \\
\hline $\begin{array}{l}\text { Anticardiolipin antibody } \\
\text { syndrome }\end{array}$ & Antithrombin deficiency \\
\hline Antiphospholipid antibodies & Prothrombin defect G20210A \\
\hline $\begin{array}{l}\text { Disseminated intravascular } \\
\text { coagulation }\end{array}$ & Dysfibrinogenemia \\
\hline Cancer and chemotherapy & Thrombomodulin defect \\
\hline Heparin thrombocytopenia & Heparinic cofactor II deficiency \\
\hline $\begin{array}{l}\text { Factor VIII, VII, I (fibrinogen) } \\
\text { and von Willebrand factor } \\
\text { increase }\end{array}$ & $\begin{array}{l}\text { Plasminogen tissue activator } \\
\text { deficiency }\end{array}$ \\
\hline Lupus anticoagulant activity & Protein S deficiency \\
\hline Hormone replacement therapy & Protein C deficiency \\
\hline Pregnancy and postpartum & Plasminogen deficiency \\
\hline Inflammatory conditions & $\begin{array}{l}\text { Excess of inhibitor } 1 \text { of } \\
\text { plasminogen activator }\end{array}$ \\
\hline $\begin{array}{l}\text { Paroxysmal nocturnal } \\
\text { hemoglobinuria }\end{array}$ & Activated protein C resistance \\
\hline Hyperhomocysteinemia & Hyperhomocysteinemia \\
\hline
\end{tabular}

supplementary tests were added to these patients during their diagnostic workup and therapeutic procedures.

\section{Patients Selection}

Between January 2005 and November 2019, 125 patients with primary idiopathic myelofibrosis preliminarily underwent US examination completed by evaluation with color Doppler and pulsed Doppler within 1 month from the diagnosis. All patients came to our observation with clinical symptoms of nonspecific abdominal pain in the superior quadrants.

Idiopathic myelofibrosis in all patients had always been diagnosed according to World Health Organization (WHO) criteria through a histological examination of the medullary puncture performed at the level of the iliac crests, use of molecular biology techniques and microscopic assessments on peripheral blood.

Among our sample (125 patients), we identified 13 patients with partial or complete portal vein thrombosis associated with portal and biliary cavernomatosis ( 5 males and 8 females, aged between 45 and 85 years).

\section{Diagnostic Imaging Techniques}

The US examination of the upper abdomen was performed by one of the authors (MS) with a supine patient after a fast of at least 6 hours to ensure a good visualization of the gallbladder and the biliary tree using two devices (Philips HD 5000 - Eindhoven, Netherlands; Esaote MyLabX6-Genua, Italy) equipped with convex probe. Scans were performed with the B-Mode technique for a morphological evaluation of the splanchnic organs by means of subcostal scans performed at the midline and at the right of the midline, both transversal and longitudinal, to visualize both the liver lobes and the gallbladder, and by means of intercostal, transverse 
and longitudinal subcostal scans to the left of the midline to visualize the spleen. The US examination was integrated with the use of color Doppler technique to evaluate caliber, course and any anomalies of the wall portal vein and suprahepatic veins. Doppler flowmetry was then performed for the dynamic sampling of the portal flow performed in inspiratory apnea.

The criteria used to diagnose biliary cavernomatosis included the detection of a concentric thickening of the walls of the MBD equal or greater than $5 \mathrm{~mm}$ and the presence of vicariant collateral venous circles within the walls of the main biliary route with demonstration of hepatopetal reduced flow rate with Doppler flowmetry.

Subsequently, all patients underwent dynamic contrast-enhanced CT scan of the abdomen with IV administration of nonionic organo-iodine contrast media. CT exams were performed using 16-detector dual MDCT equipments (Brilliance, Philips Medical System IDT, Netherlands and Lightspeed, General Electrics, USA) before and after IV administration of $120 \mathrm{ml}$ of nonionic organo-iodine contrast medium (Ultravist 300, Bayer Pharma, Germany) with an infusion rate of $3 \mathrm{~mL} / \mathrm{sec}$. Four scan acquisitions were performed on the supine patient: a precontrast phase, an early arterial phase, a portal phase, and a late phase. The contrast medium was injected into the antecubital vein through an $18 \mathrm{G}$ needle using an automatic injector (MK-IV, Medrad, Pittsburgh, Pennsylvania, USA) with 15 second (arterial phase) and 60 second (venous phase) scan delays, considering the scan time and the moment of reaching $150 \mathrm{HU}$ opacification value of the abdominal aorta (bolus tracking technique). The late equilibrium scan was acquired 180 seconds after IV administration of contrast medium.

The CT scans were performed using the following parameters: collimation $16 \times 0.75 \mathrm{~mm}$, layer thickness of $1 \mathrm{~mm}$, increment of $1 \mathrm{~mm}$, pitch 1.1, table speed $17.6 \mathrm{~mm}$, rotation time 0.5 second. and current tube $120 / 250 \mathrm{KVp} / \mathrm{mAs}$, reconstruction interval and advancement of $1 \mathrm{~mm}$. Arterial and equilibrium scans were conducted from the diaphragmatic dome to the lower pole of the kidneys; those in the portal venous phase from the diaphragmatic dome to the pubic symphysis.

At the end of the CT examination, the data obtained was transferred to a workstation (Hp Kayak XU Hewlett Packard, Palo Alto, California, USA) equipped with dedicated software (GE Centricity, USA). The images obtained were analyzed by postprocessing multiplanar reformation or reconstruction (MPR) algorithms that allowed reconstructions in the axial, coronal and sagittal planes and along oblique planes.

MR examinations were performed with 1.5 Tesla equipment (Siemens Sonata, Germany) using a phased array body coil, with the patient in supine position. The following acquisition sequences with relative scan plans have been used:

- Axial gradient-echo T1-weighted in phase and in phase opposition (TE: 5.04 milliseconds, TR: 110 milliseconds, flip angle: $70^{\circ}$, thickness: $5 \mathrm{~mm}$, number of averages: 1 ).

- Axial turbo spin echo (TSE) T2-weighted (TE: 102 milliseconds, TR: 2800 milliseconds, flip angle: $150^{\circ}$, thickness: $5 \mathrm{~mm}$, number of averages: 1 ).
- Axial TSE T2-weighted with saturation of the adipose tissue signal (TE: 102 milliseconds, TR: 2800 milliseconds, flip angle $150^{\circ}$ thickness: $5 \mathrm{~mm}$, number of averages: 1 ).

- Axial volumetric interpolated breath-hold sequences (VIBE) T1-weighted with saturation of the adipose tissue signal (TE: 1.48 milliseconds, TR: 4.3 milliseconds, flip angle: 12, thickness $4 \mathrm{~mm}$, number of averages: 1) with acquisitions made at the basal condition and repeated at 30 seconds (arterial phase), 70 seconds (portal phase) and 180 seconds (late phase) after the IV administration of $0.1 \mathrm{mmol} / \mathrm{Kg}$ of a paramagnetic contrast medium (GADOVIST, Bayer Pharma, Germany) through an 18G needle positioned in the antecubital vein with $2 \mathrm{~mL} / \mathrm{sec}$ flow rate followed by the injection of $20 \mathrm{~mL}$ of saline solution.

In four patients, MR-cholangiography study was also performed using the following acquisition sequences with the related scan plans:

- Coronal T2-weighted half-Fourier acquisition single-shot turbo spin-echo (HASTE) (TE: 122 second, TR: 699 second, flip angle: $150^{\circ}$ thickness: $3 \mathrm{~mm}$, number of averages: 1 ).

3D coronal TSE T2-weighted (TE: 681 second, TR: 4.69 second, flip angle: $170^{\circ}$, thickness: $1.5 \mathrm{~mm}$, number of averages: 1 ). These latest 3D acquisitions have been reconstructed with maximum intensity projection (MIP) technique to obtain cholangiographic-like images.

The subsequent follow-up controls in all these patients were performed only with US and MRI of the upper abdomen, avoiding $\mathrm{CT}$, to reduce radiation exposure.

\section{Statistical Analysis}

The correlation between primary myelofibrosis and the association of partial thrombosis with portal-biliary cavernoma was calculated using Chi-squared test $\left(\chi^{2}\right.$ test). Statistical analysis was performed using SPSS version 21.0 (SPSS Inc. Chicago, IL).

\section{Results}

As many as 13 out of 125 patients with known primary myelofibrosis (10\%), studied for painful abdominal symptoms, showed in the preliminary US study partial or complete thrombosis of the portal venous system and marked concentric thickening of the wall of the main biliary duct together with portal cavernomatosis. MBD thickening in these patients was between $5 \mathrm{~mm}$ and $9 \mathrm{~mm}$ (average value $7 \mathrm{~mm}$ ) (-Fig. 1). The thickening of the walls of the MBD was extended in two patients to both common hepatic ducts.

Only the use of the color Doppler technique detected within the thickened wall of the biliary tract involved the presence of ectasic serpiginous collateral veins (-Fig. 2), characterized by Doppler sampling and venous flow with hepatopetal direction and a mean average speed of approximately $15 \mathrm{~cm} / \mathrm{s}$ (-Fig. 3).

Portal vein thrombosis was partial in eight patients (62\%) and complete in five patients (38\%) and was localized at the level of the common trunk in 12 patients with extension in 10 
patients to the right portal branch and in two patients to the left portal branch. In one patient, the thrombosis was localized only to the left portal branch without any evidence of involvement of the common trunk; in this patient, the portal cavernoma was located only to the left side of the portal vein without any involvement peripheral to MBD.

The portal thrombosis never showed internal vascularity on color Doppler imaging and was always classified as a bland thrombus (-Fig. 3 ).

In 12 patients, the other US finding was the presence of thickening of the walls of the gallbladder without any intramural varices (one patient had undergone previous laparoscopic cholecystectomy), while a slight dilatation of the intrahepatic biliary tree was found in four patients. Even patients with minimal dilation of the intrahepatic biliary tracts did not have raised bilirubin or abnormal liver functions.

All 13 patients had splenomegaly with a longitudinal diameter greater than $12 \mathrm{~cm}$ with a maximum recorded value of approximately $19 \mathrm{~cm}$; in nine patients, a volumetric increase of the liver was associated without evidence by ultrasound, CT or MRI of chronic liver disease.
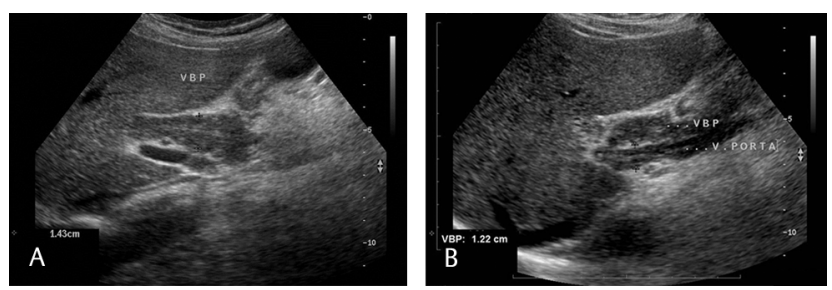

Fig. 1 (A, B) B-mode US scan performed with convex probe focused at the level of the hepatic hilum performed on two different patients with primary myelofibrosis, which demonstrates the presence of $7 \mathrm{~mm}$ concentric thickening of the main bile duct (MBD) wall in the first one (A) and of $6 \mathrm{~mm}$ in the second one (B).
Both contrast-enhanced CT and MRI confirmed the wall thickening of the biliary tract comparable to that detected on US examination (-Figs. 4, 5 and 6). Both diagnostic techniques allowed visualization of progressive enhancement of the thickened MBD walls during portal and late equilibrium phase caused by peribiliary ectasic collateral circles. Both these techniques did not allow an accurate flowmeter characterization, like the color Doppler examination, and therefore the differential diagnosis with other pathological conditions of MBD enhancing wall thickening could be difficult.

CT and MRI always confirmed the preliminary US diagnosis of portal thrombosis, allowing evaluation of its extension with greater accuracy (partial or complete thrombosis) and demonstrating the involvement of splenic vein in one patient and superior mesenteric vein in two patients.

Both contrast-enhanced CT and MRI allowed detection of the presence of areas of liver parenchyma increase density during the arterial dynamic phase (transient hepatic attenuation differences [THAD]) in nine patients and the presence of multiple perigastric and perisplenic vicariant venous collateral circles in four patients.

In the four patients studied with MR-cholangiography, 3D coronal acquisitions and MIP reconstructions, a filiform aspect of the main biliary duct was always detected associated with minimal ab extrinsic compression but without the detection of a sudden interruption of the lumen caliber (-Fig. 6).

In our study the concurrent occurrence of simultaneous portal thrombosis with portal-biliary cavernomatosis in patients with primary myelofibrosis was observed in 13 cases. This result implies a "Chi squared" value $\chi^{2}=0$ that confirms the coexistence of portal thrombosis together with portal-biliary cavernoma in our patients.
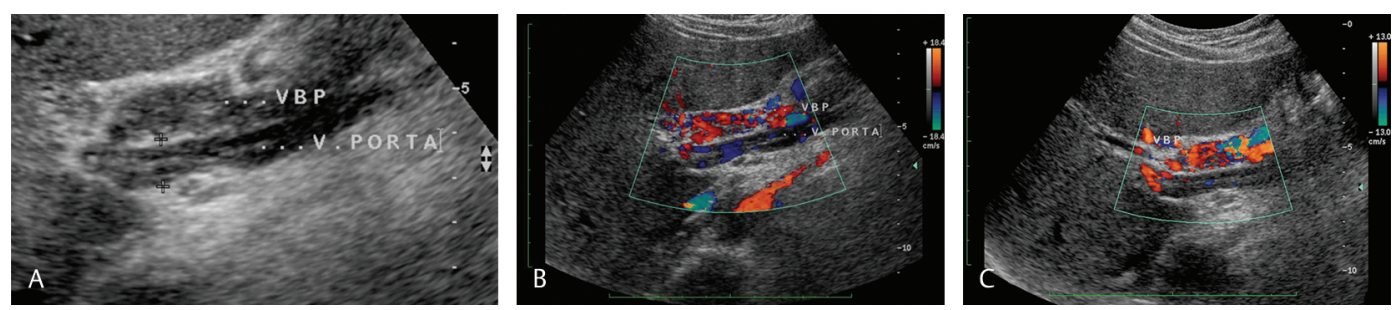

Fig. 2 (A-C) B-mode US scan performed on a patient with primary myelofibrosis, which demonstrates the presence of a $7 \mathrm{~mm}$ concentric thickening of the main bile duct (MBD) wall (A). Color Doppler showed the absence of flow within the portal lumen due to a complete thrombosis and the evidence of flow within the MBD wall thickened due to collateral venous vessels (B, C).
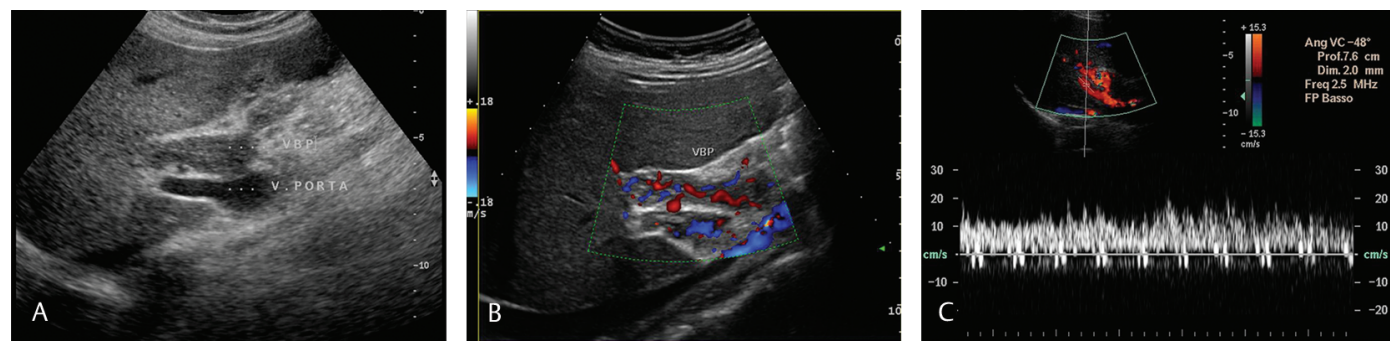

Fig. 3 (A-C) B-mode US scan performed on patient with primary myelofibrosis, which shows $6 \mathrm{~mm}$ concentric thickening of the main bile duct (MBD) wall and a partial portal thrombosis $(\mathbf{A}, \mathbf{B})$. Color Doppler $(\mathbf{B})$ with flow-velocity sampling $(\mathbf{C})$ of the vessels within the MBD wall was performed confirming the definitive diagnosis of biliary cavernoma. 


\section{Discussion}

Our experience confirmed the possibility of a close association between portal thrombosis and cavernomatous transformation of the biliary tract in patients with primary myelofibrosis, ${ }^{19-21}$ which was found in $10 \%$ of our patients. All thirteen patients with primary myelofibrosis observed for portal system thrombosis also developed portal and biliary cavernomatosis. Biliary cavernomatosis is rather unusual and uncommon compared with the more known and frequent portal cavernomatosis.

Myelofibrosis is a rare chronic myeloproliferative disease recognized in two forms, the idiopathic or primary form and the secondary form which represents the late evolution of a pre-existing true polycythemia or essential thrombocythemia. It consists of a clonal disease of the hematopoietic stem cell characterized by megakaryocyte and myeloid proliferation, a reactive medullary fibrosis with consequent ineffective erythropoiesis and extramedullary
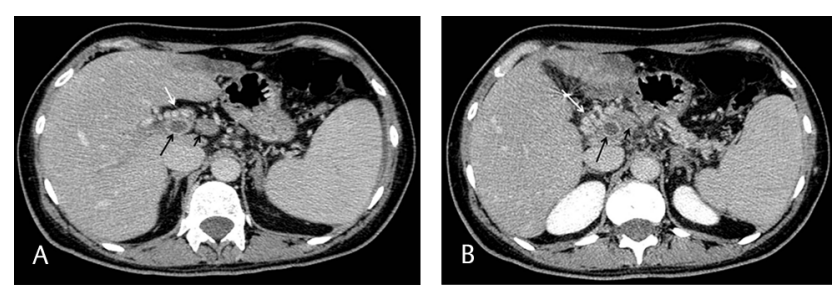

Fig. 4 (A, B) Multi-detector computed tomography (MDCT) scans performed at the level of the hepatic hilum on two different planes (A, B) after the IV administration of contrast media during the portal phase. It is possible to demonstrate the portal vein thrombosis (short black arrow), a concentric thickening of the main bile duct (MBD) wall with contrast enhancement due to biliary cavernoma (long black arrow) and the presence of numerous dilated collateral periportal vessels due to portal cavernoma (white arrow).
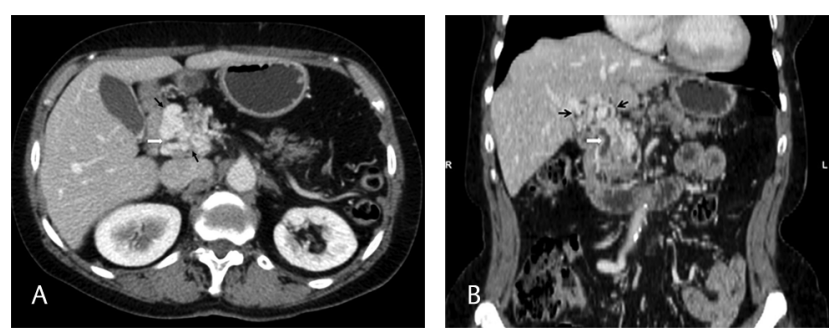

Fig. 5 (A, B) Multi-detector computed tomography (MDCT) scans performed at the level of the hepatic hilum on axial plane (A) and a multiplanar reformatting on coronal plane (B) after the IV administration of contrast media during the portal phase. It is possible to demonstrate the normal caliber of the main bile duct (MBD) (white arrow) and the presence of numerous dilated collateral periportal vessels due to portal-biliary cavernoma (black arrows), which is better detectable on axial plane. hematopoiesis. ${ }^{17,18}$ The diagnosis of primary myelofibrosis, according to WHO, is based on histopathological, molecular and clinical criteria, and histopathology represents the key in the diagnostic process with the demonstration of atypia in the proliferation of megakaryocytes. ${ }^{22,23}$ The most frequent clinical manifestations are anemia, asthenia, hepatosplenomegaly, extramedullary hematopoiesis and thrombohemorrhagic complications. ${ }^{16,17,24}$ Among myeloproliferative diseases, idiopathic myelofibrosis has the worst prognosis in terms of both survival and quality of life with an average survival of approximately 69 months from the time of diagnosis reported. The main causes of death are the evolution of acute myeloid leukemia, occurrence of an accelerated non-leukemic phase, recurrent infections and thrombosis. ${ }^{24}$

Denys et $\mathrm{al}^{7}$ found an association between myelofibrosis and cavernomatous transformation of the portal system and biliary tree, and our study appears to support the correlation between these two pathologies. The cause of this association with primary myelofibrosis is probably due to the gradual formation of portal vein thrombosis, which is related to hypercoagulability status. This would result in a slow development of portal and biliary cavernomatosis, even in patients with partial portal vein thrombosis. The portal thrombosis was always due to a bland thrombus (nonmalignant venous thrombus) which appear avascular on color Doppler. The differential diagnosis is very important for the therapeutic approach because bland thrombus may resolve after thrombolytic and anticoagulant therapy, unlike tumor thrombus.

Our experience also shows that cavernomatosis of the main biliary tract is not a rare and unusual condition as suggested by the available literature, but is probably a more frequent condition often confused with other causes of parietal thickening of the biliary tree and gallbladder., ${ }^{6,25}$

The thickness of the MBD wall found was always between $5 \mathrm{~mm}$ and $9 \mathrm{~mm}$. These values confirm the work of Denys et $\mathrm{al}^{7}$ in which the three patients showed wall thickening between $5 \mathrm{~mm}$ and $6 \mathrm{~mm}$. Thickening of the MBD equal or greater than $5 \mathrm{~mm}$ can be considered compatible with the possible diagnosis of biliary cavernomatosis. In this regard, the thickening of the MBD and common hepatic ducts found on B-mode US examination in patients with portal vein thrombosis could be incorrectly attributed to various pathological conditions such as chronic cholangitis and Klatskin's tumor rather than a cavernous transformation of the portal and biliary spaces. ${ }^{6,7}$ In our study, B-mode US proved to be completely ineffective in identifying the nature of the concentric thickening of the biliary tract walls; only the color Doppler allowed to detect the presence of serpiginous vessels with hepatopetal venous flow within the wall of the biliary
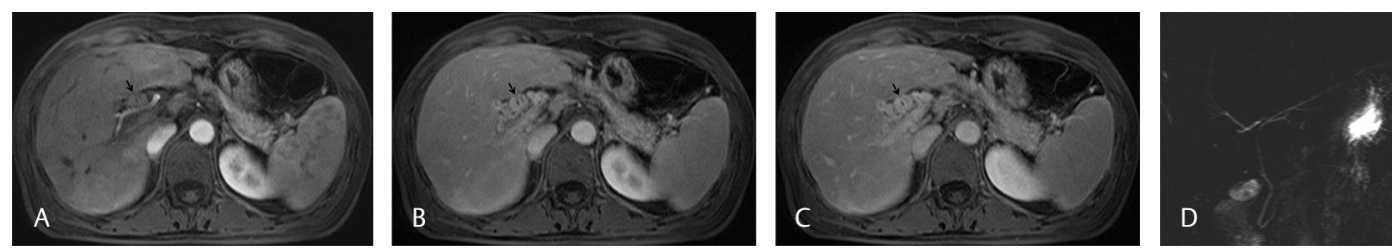

Fig. 6 (A-D) Axial contrast-enhanced MRI performed in a patient with primary myelofibrosis, portal thrombosis and biliary cavernoma at the level of the hepatic hilum during the arterial (A), portal (B) and late phase (C), which detected progressive enhancement of the main bile duct (MBD) thickened wall (black arrow). MR-cholangiography (D) shows the normal caliber of the MBD. 
tract; in our opinion, this is the pathognomonic US sign of biliary cavernomatosis.

Compared with the US, MDCT and MRI allowed better assessment of the extent of venous thrombosis and the morphological aspect of the biliary system and usually permitted exclusion of an obstruction of the main biliary duct. MRI with MR-cholangiography are particularly accurate in the evaluation of the biliary tract lumen and in the differential diagnosis with malignant conditions of MBD parietal thickening, allowing to exclude the presence of tight stenosis suggestive of malignancy.

According to Aguirre et al, ${ }^{26}$ and in our experience, in biliary cavernomatosis, MR-cholangiography demonstrates a threadlike aspect of MBD with signs of ab extrinsic compression and without the finding of significative caliber reduction.

In conclusion, the constant finding in primary myelofibrosis of the association of portal thrombosis with portal-biliary cavernoma indicates the existence of a correlation between these two pathologies. For the detection of the ectasic vessels developing from the vasa vasorum of the portal vein and biliary tract, characteristic of the cavernomatous conditions, the most accurate diagnostic technique is represented by US with color Doppler, which allows one to make a definitive diagnosis, without using more expensive techniques and contrast media. ${ }^{3} \mathrm{CT}$ and/or MRI must be used to evaluate the extent of portal thrombosis, to follow-up thrombolytic therapy and correctly study the manifestations and complications of the underlying disease such as spleen involvement and extramedullary hematopoiesis.

\section{Conflict of Interest}

None declared.

\section{Acknowledgments}

The authors would like to thank Prof. James Marsh Sternberg, M.D. for some valuable suggestions for this manuscript, Prof. Paolo Di Girolamo, PhD, for assistance in the statistical analysis, and Prof. Andrea Laghi, M.D., who was the guarantor of our study.

\section{References}

1 Vibert E, Azoulay D, Castaing D, Bismuth H. [Portal cavenorma: diagnosis, aetiologies and consequences]. Ann Chir 2002;127(10):745-750

2 De Gaetano AM, Lafortune M, Patriquin H, De Franco A, Aubin B, Paradis K. Cavernous transformation of the portal vein: patterns of intrahepatic and splanchnic collateral circulation detected with Doppler sonography. AJR Am J Roentgenol 1995;165(5):1151-1155

3 Grgurević I, Buljevac M, Kujundzić M, Vukelić-Marković M, Kardum D, Brkljacić B. Common bile duct wall thickening due to intramural varices diagnosed by colour Doppler ultrasound. Ultraschall Med 2006;27(5):483-486

4 Saint JH. The epicholedochal venous plexus and its importance as a means of identifying the common duct during operations on the extrahepatic biliary tract. Br J Surg 1961;48:489-498

5 Hunt AH. Compression of the common bile-duct by an enlarging collateral vein in a case of portal hypertension. Br J Surg 1965;52:636-637

6 Novellas S, Chevallier P, Peroux JL, Bruneton JN. Rare localization of a portal cavernoma in the wall of the common bile duct. Clin Imaging 2004;28(2):132-134
7 Denys $\mathrm{A}$, Hélénon $\mathrm{O}$, Lafortune $\mathrm{M}$, et al. Thickening of the wall of the bile duct due to intramural collaterals in three patients with portal vein thrombosis. AJR Am J Roentgenol 1998;171(2):455-456

8 Gupta S, Singhal A, Goyal N, Vij V, Wadhawan M. Portal biliopathy treated with living-donor liver transplant: index case. Exp Clin Transplant 2011;9(2):145-149

9 Agarwal AK, Sharma D, Singh S, Agarwal S, Girish SP. Portal biliopathy: a study of 39 surgically treated patients. HPB (Oxford) 2011;13(1):33-39

10 Chow L, Jeffrey RB Jr. Intramural varices of the bile duct: an unusual pattern of cavernous transformation of the portal vein. AJR Am J Roentgenol 1999;173(5):1255-1256

11 Besa C, Cruz JP, Huete A, Cruz F. Portal biliopathy: a multitechnique imaging approach. Abdom Imaging 2012;37(1):83-90

12 DeLeve LD, Valla DC, Garcia-Tsao G; American Association for the Study Liver Diseases. Vascular disorders of the liver. Hepatology 2009;49(5):1729-1764

13 Diaz E, Nahon S, Charachon A, et al. [Portal vein thrombosis associated with a myeloproliferative disorder, prothrombin G20210A mutation, antiphospholipid syndrome, with repermeation during anticoagulant therapy]. Gastroenterol Clin Biol 2001;25(5):549-551

14 Xavier SG, Gadelha T, Pimenta G, et al. JAK2V617F mutation in patients with splanchnic vein thrombosis. Dig Dis Sci 2010;55(6):1770-1777

15 Denninger MH, Chaït Y, Casadevall N, et al. Cause of portal or hepatic venous thrombosis in adults: the role of multiple concurrent factors. Hepatology 2000;31(3):587-591

16 Saliba W, Mishchenko E, Cohen S, Rennert G, Preis M. Association between myelofibrosis and thromboembolism: A population-based retrospective cohort study. J Thromb Haemost 2020;18(4):916-925

17 Gregory SA, Mesa RA, Hoffman R, Shammo JM. Clinical and laboratory features of myelofibrosis and limitations of current therapies. Clin Adv Hematol Oncol 2011;9(9, Suppl 22):1-16

18 DeLario MR, Sheehan AM, Ataya R, et al. Clinical, histopathologic, and genetic features of pediatric primary myelofibrosis-an entity different from adults. Am J Hematol 2012;87(5):461-464

19 Barbui T, Carobbio A, Cervantes F, et al. Thrombosis in primary myelofibrosis: incidence and risk factors. Blood 2010;115(4):778-782

20 Cervantes F, Alvarez-Larrán A, Arellano-Rodrigo E, Granell M, Domingo A, Montserrat E. Frequency and risk factors for thrombosis in idiopathic myelofibrosis: analysis in a series of 155 patients from a single institution. Leukemia 2006; 20(1):55-60

21 Ogawa S, Doishita K, Yamazaki Y, Nakanuma Y, Ohta G. Primary myelofibrosis associated with portal venous thrombotic occlusion and cavernous transformation around the biliary tree. J Clin Gastroenterol 1987;9(1):115-116

22 Hamidah NH, Farisah NR, Azlinda AB, et al. A study of JAK2 (V617F) gene mutation in patients with chronic myeloproliferative disorders. Clin Ter 2012;163(2):109-113

23 Thiele J, Kvasnicka HM, Zankovich R, Diehl V. Relevance of bone marrow features in the differential diagnosis between essential thrombocythemia and early stage idiopathic myelofibrosis. Haematologica 2000;85(11):1126-1134

24 Vannucchi AM. Management of myelofibrosis. Hematology (Am Soc Hematol Educ Program) 2011;2011:222-230

25 Chandra R, Kapoor D, Tharakan A, Chaudhary A, Sarin SK. Portal biliopathy. J Gastroenterol Hepatol 2001;16(10):1086-1092

26 Aguirre DA, Farhadi FA, Rattansingh A, Jhaveri KS. Portal biliopathy: imaging manifestations on multidetector computed tomography and magnetic resonance imaging. Clin Imaging 2012;36(2):126-134 